\title{
(C) OPEN ACCESS \\ Dysregulation of the intrinsic apoptotic pathway mediates megakaryocytic hyperplasia in myeloproliferative neoplasms
}

\author{
Jacques A J Malherbe, ${ }^{1}$ Kathryn A Fuller, ${ }^{1,2}$ Bob Mirzai, ${ }^{1,2}$ Simon Kavanagh, ${ }^{1,2}$ \\ Chi-Chiu So, ${ }^{3}$ Ho-Wan Ip, ${ }^{4}$ Belinda B Guo, ${ }^{1}$ Cecily Forsyth, ${ }^{5}$ Rebecca Howman, ${ }^{2}$ \\ Wendy $N$ Erber $^{1,2}$
}

${ }^{1}$ School of Pathology and Laboratory Medicine, University of Western Australia, Crawley, Western Australia, Australia ${ }^{2}$ PathWest Laboratory Medicine, Nedlands, Western Australia, Australia ${ }^{3}$ Department of Pathology, Faculty of Medicine, University of Hong Kong, Hong Kong, Hong Kong

${ }^{4}$ Department of Pathology \& Clinical Biochemistry, Queen Mary Hospital, Hong Kong, Hong Kong

${ }^{5}$ Jarrett Street Specialist Centre, North Gosford, New South Wales, Australia

\section{Correspondence to} Professor Wendy N Erber, School of Pathology and Laboratory Medicine (M504), The University of Western Australia: 35 Stirling Highway, Crawley, Western Australia 6009, Australia wendy.erber@uwa.edu.au

Received 14 January 2016 Revised 8 March 2016 Accepted 10 March 2016 Published Online First 8 April 2016

\begin{abstract}
Aims Megakaryocyte expansion in myeloproliferative neoplasms (MPNs) is due to uncontrolled proliferation accompanied by dysregulation of proapoptotic and antiapoptotic mechanisms. Here we have investigated the intrinsic and extrinsic apoptotic pathways of megakaryocytes in human MPNs to further define the mechanisms involved.

Methods The megakaryocytic expression of proapoptotic caspase-8, caspase-9, Diablo, p53 and antiapoptotic survivin proteins was investigated in bone marrow specimens of the MPNs $(n=145)$ and controls $(n=15)$ using immunohistochemistry. The megakaryocyte percentage positivity was assessed by light microscopy and correlated with the MPN entity, JAK2 ${ }^{\mathrm{V} 617 \mathrm{~F}} / \mathrm{CALR}$ mutation status and platelet count.
\end{abstract}

Results The proportion of megakaryocytes in the MPNs expressing caspase-8, caspase-9, Diablo, survivin and p53 was significantly greater than controls. A greater proportion of myeloproliferative megakaryocytes expressed survivin relative to its reciprocal inhibitor, Diablo. Differences were seen between myelofibrosis, polycythaemia vera and essential thrombocythaemia for caspase-9 and p53. CALR-mutated cases had greater megakaryocyte p53 positivity compared to those with the JAK2 ${ }^{\mathrm{V} 617 \mathrm{~F}}$ mutation. Proapoptotic caspase-9 expression showed a positive correlation with platelet count, which was most marked in myelofibrosis and CALR-mutated cases.

Conclusions Disruptions targeting the intrinsic apoptotic cascade promote megakaryocyte hyperplasia and thrombocytosis in the MPNs. There is progressive dysfunction of apoptosis as evidenced by the marked reduction in proapoptotic caspase- 9 and accumulation of p53 in myelofibrosis. The dysfunction of caspase-9, which is necessary for proplatelet formation, may be the mechanism for the excess thrombocytosis associated with CALR mutations. Survivin seems to be the key protein mediating the megakaryocyte survival signature in the MPNs and is a potential therapeutic target.

\section{INTRODUCTION}

CrossMark

To cite: Malherbe JAJ, Fuller KA, Mirzai B, et al. J Clin Pathol 2016;69:10171024.
Myeloproliferative neoplasms (MPNs) are a group of clonal proliferative bone marrow diseases characterised by somatic mutations (eg, JAK2 ${ }^{\mathrm{V} 617 \mathrm{~F}}, C A L R$ frameshift lesions) ${ }^{1-5}$ and varying hyperplasia of the myeloid lineages. Megakaryocyte hyperplasia with clustering and associated morphological atypia with pleiomorphism are key diagnostic histological features. ${ }^{6-8}$ The pathobiological basis underlying these numerical and morphological megakaryocytic abnormalities is thought to result from multiple molecular disruptions promoting proliferation and enhancing survival. ${ }^{9-13}$ These megakaryocytes have impaired death mechanisms conferred by overexpression of antiapoptotic Bcl-XL and reductions in pro-death BNIP-3. ${ }^{911-13}$ These changes are universal in the MPNs but there are differences between entities. Megakaryocytes in essential thrombocythaemia (ET) have been shown to have a more proliferative profile, whereas in myelofibrosis (MF) they exhibit greater proapoptotic impairments. ${ }^{9} 13$ These changes occur irrespective of the $J A K 2^{\mathrm{V} 617 \mathrm{~F}}$ or calreticulin $(C A L R)$ driver mutations, although those with a CALR lesion have greater proapoptotic dysfunction. ${ }^{13}$ The mechanisms driving this apoptotic dysregulation in megakaryocytes in the MPNs have not been explored.

Apoptosis is mediated via extrinsic and intrinsic apoptotic cascades (figure 1), with both pathways being capable of inducing programmed cell death following exposure to apoptotic insults and the accumulation of excess DNA damage. ${ }^{14-16}$ These pathways converge at the cleavage of procaspase- 3 to produce active caspase-3, which is capable of committing the cell to apoptosis. ${ }^{14-16}$ Alterations in caspase biology, including caspase- 8 (extrinsic) and caspase-9 (intrinsic), have been implicated in a number of malignancies in humans and animal models. In caspase-8-deficient mice, B-lymphocytes have impaired cytokinesis and chromosomal instability, and show a tendency towards lymphoma development. ${ }^{17}$ Similarly, caspase- 9 gene polymorphisms and its downregulation are associated with solid tumours and their malignant progression. ${ }^{18-20}$ Both caspase- 8 and caspase- 9 are potentially important in regulating megakaryocyte turnover in the MPNs. Frameshift lesions targeting CALR may disrupt megakaryocyte apoptosis through its inability to facilitate caspase- 8 activation and antiapoptotic protein cleavage. ${ }^{21}$ While the precise pathogenesis surrounding CALR lesions and megakaryocyte apoptosis is unknown, its mutated product is exclusively expressed in myeloproliferative megakaryocytes. ${ }^{22} 23$ In megakaryocytes, caspase-9 activity appears necessary for proplatelet formation. ${ }^{24}$ Some reports suggest that caspase- 9 may be redundant, with CASP9 deletions in ex vivo-cultured mouse megakaryocytes impairing procaspase-3 activation, and 


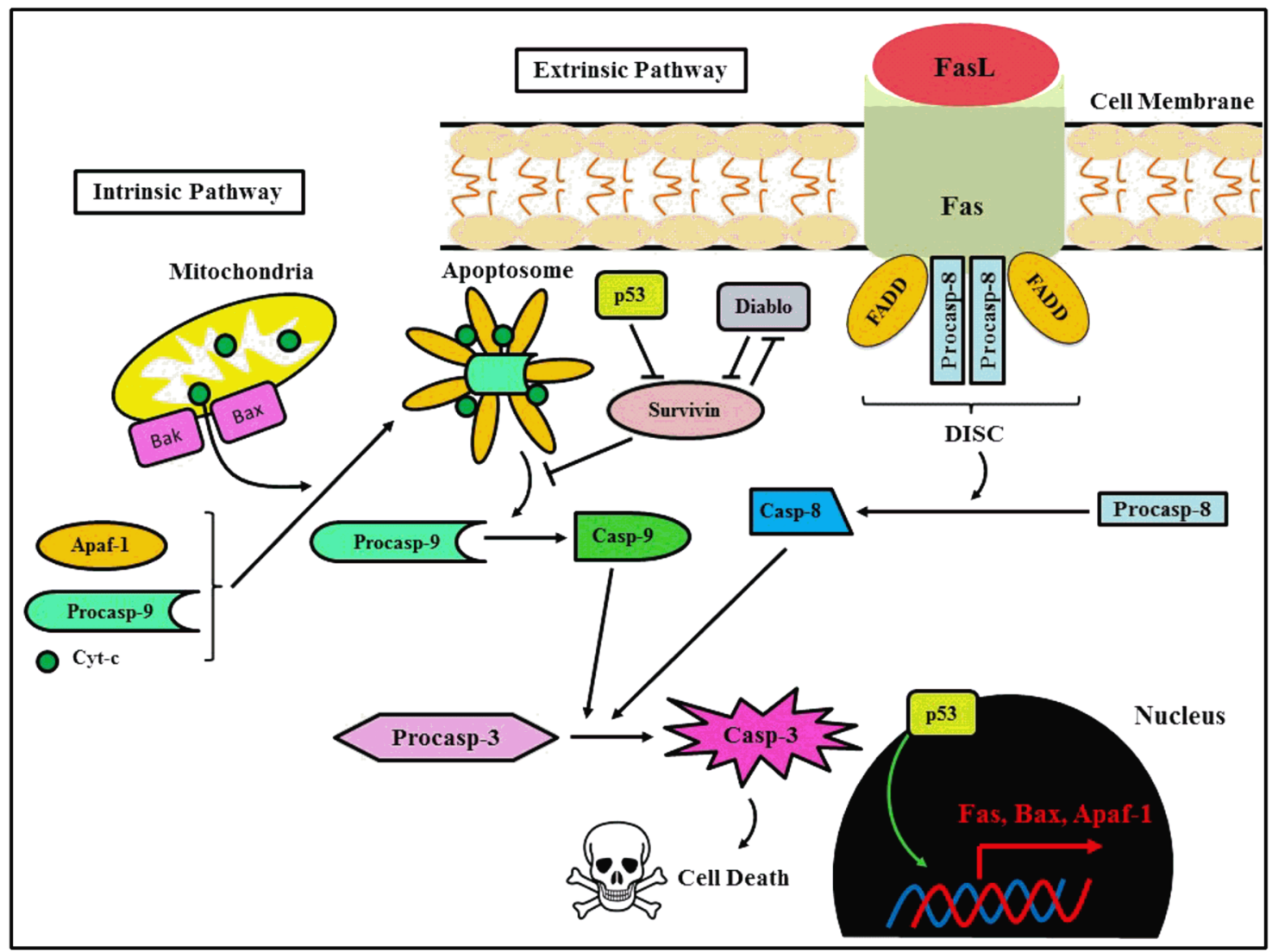

Figure 1 Diagrammatic representation of the extrinsic and intrinsic apoptotic pathways. The extrinsic (receptor-mediated) apoptotic pathway commences following FasL binding the Fas death receptor. The union forms a death complex that is capable of recruiting FADD and procaspase- 8 to form the DISC. The DISC recruits procaspase-8 proteins that undergo reciprocal cross-proteolysis and proximity-induced dimerisation to form an active caspase-8 that targets procaspase-3. The intrinsic (mitochondrial-mediated) apoptotic pathway relies on the insertion of homo-oligomerised pro-death Bcl-2-family proteins (Bak and Bax) into the outer mitochondrial membrane. The release of cytochrome-c subsequently ensues and acts to recruit procaspase- 9 and Apaf-1 to form the heptameric apoptosome. The apoptosome recruits procaspase- 9 molecules, which reciprocally cleave one another to form an active caspase- 9 that targets procaspase-3. Both caspase- 8 and caspase- 9 then cleave procaspase- 3 to form the executioner caspase-3, which brings about coordinated cell death. Survivin aims to inhibit caspase- 9 activation while simultaneously competing with the inhibitory action of cytosolic p53 and Diablo. Nuclear p53 also activates the transcription of pro-death molecules required in both apoptotic cascades. Apaf-1, apoptotic protease activating factor-1; Bak, Bcl-2 homologous antagonist/killer; Bax, Bcl-2-associated X protein; Casp-3,

caspase-3; Casp-8, caspase-8; Casp-9, caspase-9; Cyt-c, cytochrome-c; DISC, death-inducing signalling complex; FADD, Fas-associated protein with death domain; Fas, Fas death receptor; FasL, Fas receptor ligand; Procasp-3, procaspase-3; Procasp-8, procaspase-8; Procasp-9, procaspase-9.

encouraging both megakaryopoiesis and proplatelet formation. ${ }^{25}$ This does not however discount the presence of other caspases to conduct cytoskeletal proplatelet fragmentation in the absence of caspase-9. Moreover, caspase-9 loss may enhance the tumourigenic potential of megakaryocytes as their proliferative capacity increases. ${ }^{25}$

Apoptotic cell death via the intrinsic pathway is regulated by inhibitors of apoptosis proteins (IAP) that specifically constrain the pro-death actions of caspase-9. One of these IAP, survivin, restricts the IAP-inhibitor Diablo protein and prevents it from activating caspase-9. ${ }^{26-29}$ This impedes the intrinsic apoptotic pathway and confers a cytoprotective effect. The relevance of Diablo has been shown in human tumours: downregulation has been associated with progressive disease and poor survival in both solid and haematological malignancies. ${ }^{30-33}$ Survivin overexpression is well documented in leukaemias ${ }^{34-37}$ and lymphomas $^{38-41}$ where it increases the survival capacity of affected tumour cells. On the other hand, survivin loss causes mitotic catastrophe characterised by cell death or polyploidisation. ${ }^{37} 42$ Mouse megakaryocytes failing to express survivin have limited proliferation but increased ploidy, with loss of the IAP preferentially selecting for those megakaryocytes capable of escaping intrinsic apoptotic fates. ${ }^{42}$ Megakaryocytes are known to express survivin and during anaphase it restricts cytokinesis to enable polyploidisation. ${ }^{43}$ The relationship between survivin, Diablo and caspase- 9 is complex and has not been assessed in megakaryocyte survival in the MPNs.

p53, the 'master' regulator of cell cycle, is also involved in the apoptotic machinery. Its regulatory role involves arresting the cell cycle, initiating senescence and inducing DNA repair mechanisms. ${ }^{44}$ Failing these, p53 activates the intrinsic apoptotic cascade by binding Bak/Bax to induce cytochrome-c release while simultaneously inhibiting antiapoptotic Bcl-2-related proteins ${ }^{46} 47$ and survivin. ${ }^{48}$ The role of p53 in megakaryocytes is disputed. Ex vivo-cultured megakaryocytes have been shown to express low levels of p53 ${ }^{49}$ and its absence in p53-null mice has no effect on megakaryopoiesis. ${ }^{50}$ However, the in vitro knockdown of p53 has been reported to increase megakaryocyte proliferation and regulate both its ploidy and differentiation. ${ }^{51-54}$ This disparity between in vitro and ex vivo experimental settings ultimately suggests that in vivo, p53 loss is tolerated in the presence of other compensatory proapoptotic mechanisms. In the 
MPNs, alterations in $\mathrm{p} 53$ have not been linked to megakaryocyte hyperplasia although mutations targeting TP53 do occur during their leukaemic transformation. ${ }^{55-57}$

Apoptotic signalling is crucial to megakaryocyte polyploidisation and platelet production. Limited studies have identified that apoptotic signalling processes are disrupted in megakaryocytes of the MPNs. ${ }^{9-13}$ We intended to further delineate the biological basis of the apoptotic disturbances affecting megakaryocytes in the MPNs by assessing several biomarkers implicated in the intrinsic and extrinsic apoptotic pathways. We demonstrate through immunohistochemical analyses of human MPNs that the enhanced survival of myeloproliferative megakaryocytes occurs through inhibition of intrinsic death effectors despite concurrent extrinsic apoptotic activation.

\section{MATERIALS AND METHODS \\ Patient samples}

Bone marrow trephine (BMT) specimens from patients with polycythaemia vera (PV), ET and MF (including both primary $\mathrm{MF}$ and post-PV and post-ET MF) $(\mathrm{n}=145)$ and normal bone marrow controls $(n=15)$ were collected from patients through PathWest Laboratory Medicine (Western Australia, Australia) and Queen Mary Hospital (Hong Kong SAR, China) between 1999 and 2015. Of the 145 patients with MPNs, 133 were untreated at the time of bone marrow collection. The PathWest specimens were formalin-mercury fixed while those from Hong Kong were fixed in formalin. All BMT specimens were acid decalcified and paraffin embedded. Morphological review of all cases was undertaken in accordance with WHO criteria ${ }^{7}$ and classified according to MPN entity (table 1). PathWest BMT specimens were processed using a TMA Master tissue microarrayer (3DHistech, Australia) to create tissue microarrays (TMA) as outlined in Malherbe et al. ${ }^{13}$ Whole BMT sections from the Hong Kong cohort were used for immunohistochemical investigation. JAK2 ${ }^{\mathrm{V} 617 \mathrm{~F}}$ mutation testing was performed by allelespecific PCR analysis. CALR mutations were detected using methods outlined by Nangalia et al. ${ }^{5}$ MPL mutation testing was not performed and cases negative for JAK2 ${ }^{\mathrm{V} 617 \mathrm{~F}}$ and CALR mutations were classified as 'double negative' (DN). Platelet counts $\left(\times 10^{9} / \mathrm{L}\right)$ were recorded for each MPN case at the time of collection of the BMT specimen.

\section{Immunohistochemical staining}

Whole BMT specimens and TMA were sectioned at $4 \mu \mathrm{m}$ onto charged glass slides (Hurst Scientific, Australia). Monoclonal antibodies were to formalin-mercury-resistant epitopes and were

Table 1 Summary of the cohort studied

\begin{tabular}{|c|c|c|c|c|c|}
\hline \multirow[b]{2}{*}{ Subtype } & \multicolumn{4}{|c|}{$J A K 2^{\mathrm{V} 617 \mathrm{~F}}$ and $C A L R$ mutation status } & \multirow[b]{2}{*}{ Total } \\
\hline & $J A K 2^{\mathrm{V} 617 \mathrm{~F}+}$ & CALR $R^{\mathrm{Mut}}$ & $J A K 2^{\mathrm{V} 617 \mathrm{~F}-} / C A L R^{\mathrm{WT}}$ & Unknown & \\
\hline Controls & - & - & 15 & - & 15 \\
\hline PV & 20 & - & - & - & 20 \\
\hline ET & 26 & 9 & 6 & 31 & 72 \\
\hline $\mathrm{MF}^{*}$ & 18 & 14 & 5 & 16 & 53 \\
\hline Total & 64 & 23 & 26 & 47 & 160 \\
\hline
\end{tabular}

validated on control tissue prior to their application on BMT sections. Antibodies used were to CD61 (clone 2f2, Leica Biosystems, Australia), caspase-8 (clone 90A992, Thermo Scientific Pierce Antibodies, Australia), caspase-9 (clone F-7, Santa Cruz Biotechnology, USA), p53 (clone DO-7, Leica Biosystems, Australia), survivin (clone 71G4B7, Cell Signaling Technologies, USA) and Diablo/Smac (clone D5S3R, Cell Signaling Technologies, USA). All immunohistochemical staining was performed on an automated Leica BOND RX immunostainer (Leica Biosystems) as outlined by Malherbe et al. ${ }^{13}$ Positive and negative megakaryocytes were counted by a minimum of two observers and the percent positive calculated for each apoptotic biomarker. Megakaryocyte-rich $(\geq 50)$ areas within whole BMT specimens were selected for enumeration of each biomarker up to a maximum of 200 megakaryocytes. Observers were blinded to both the diagnostic entity and mutation status of cases. Tissue areas and/or megakaryocytes of interest were photographed using a Pixera Pro 600ES microscope camera (Pixera, USA).

\section{Statistical analysis}

Mean megakaryocyte percentage positivity and SD were calculated according to the MPN subtype and $J A K 2^{\mathrm{V} 617 \mathrm{~F}} / C A L R$ mutational status for each apoptotic biomarker. Significant differences between the MPNs and control megakaryocyte positivity were assessed using Mann-Whitney $U$ tests. One-way Kruskal-Wallis analysis of variance (ANOVA) analyses with post hoc Dunn's tests were performed to evaluate megakaryocyte positivity variations in relation to subtype and $J A K 2^{\mathrm{V} 617 \mathrm{~F}} /$ CALR mutation status. Platelet counts for the MPNs stratified according to subtype and JAK2 ${ }^{\mathrm{V} 617 \mathrm{~F}} / C A L R$ mutation status were correlated with the mean megakaryocyte expression for all biomarkers using Spearman's correlation. Significant differences were set at $\mathrm{p}<0.05$. All statistical analyses were conducted using GraphPad Prism V.6 software (GraphPad Software, USA).

\section{RESULTS}

\section{MPNs versus controls}

Megakaryocytes were visually identified on morphology, antigen expression (red chromogen) and nuclear haematoxylin counterstain. All antibodies produced the expected expression profile; there was no non-specific background staining in any sections. CD61 was used to confirm the identity of megakaryocytes in BMT with marked atypia (eg, MF). Mean megakaryocyte positivity was significantly greater in the MPNs than in controls for all apoptotic biomarkers, that is, caspase-8, caspase-9, survivin, Diablo and p53 (figure 2). Caspase-8 showed significantly more $(\sim 16.8 \%)$ positive megakaryocytes in the MPNs than controls, $\mathrm{p}=0.0005$. A similar significant trend was observed for caspase-9, although this increase was smaller ( 4.3\%), $\mathrm{p}=0.023$. Of note was that megakaryocytes present within clusters, a key feature of the MPNs, showed strong positivity for both caspase- 8 and caspase-9, whereas single intertrabecular or paratrabecular megakaryocytes were more commonly negative (figure 3A-C). There were significantly greater numbers of Diablo and survivin-positive megakaryocytes in the MPNs than controls, $\sim 3.1$-fold, $\mathrm{p}<0.0001$ and $\sim 1.8$-fold, $\mathrm{p}<0.0001$, respectively. When comparing the proportion of Diablo and survivin-positive megakaryocytes in the MPNs, the balance was in favour of survivin by $\sim 19.0 \%$. The cytoplasmic expression of Diablo was weak and heterogeneous among megakaryocyte clusters in the MPNs (figure 3D). Contrastingly, myeloproliferative megakaryocytes showed strong nuclear localisation of survivin 
Figure 2 Percentage of positive megakaryocytes in myeloproliferative neoplasms (MPNs) and controls for and p53. Mean megakaryocyte positivity in MPN cases was significantly greater for all biomarkers in comparison to controls. Statistically significant difference, $\mathrm{p}<0.05\left({ }^{*}\right)$, $\mathrm{p}<0.001\left(^{* * *}\right)$ caspase-8, caspase-9, Diablo, survivin

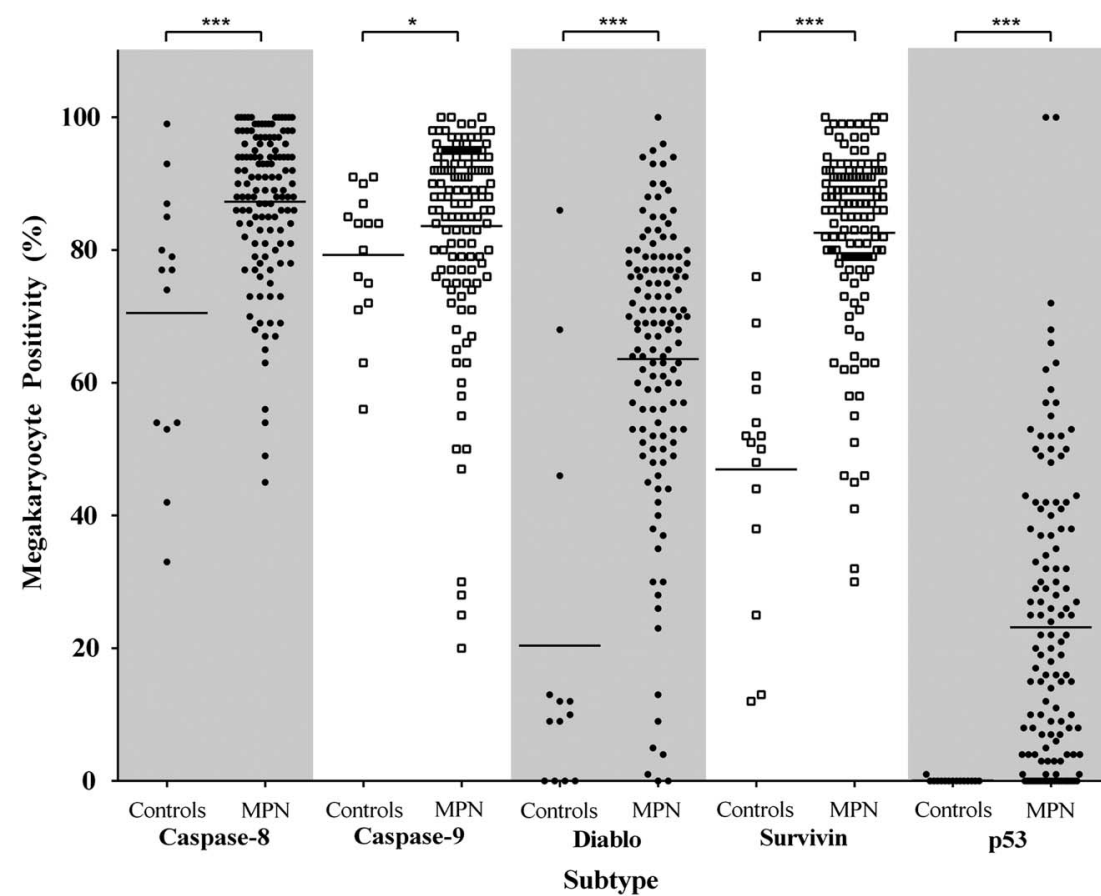

(figure 3E, F). p53 was positive (nuclear) in myeloproliferative megakaryocytes $(\sim 23.1 \%)$ and virtually absent from controls, $\mathrm{p}<0.0001$ (figure $3 \mathrm{G}, \mathrm{H}$ ).

\section{MPNs by disease entity and genotype}

Since myeloproliferative megakaryocytes showed increased expression of apoptosis-associated antigens, we then proceeded to assess reactivity by morphological entity (ie, PV, ET and MF) and mutation status (ie, JAK2 $2^{\mathrm{V} 617 \mathrm{~F}+}, C A L R^{\mathrm{Mut}}$ and $\mathrm{DN}$ : $\left.J A K 2^{\mathrm{V} 617 \mathrm{~F}^{-}} / C A L R^{\mathrm{WT}}\right)$. Using Kruskal-Wallis ANOVA analyses, significant differences were seen between the MPN subtypes for caspase- 9 and $\mathrm{p} 53, \mathrm{p}=0.0015$ and $\mathrm{p}=0.0079$, respectively (figure 4). Post hoc analyses showed caspase-9 expression to be significantly lower in MF megakaryocytes ( 77.9\%) than both PV $(\sim 90.3 \%, \mathrm{p}=0.0032)$ and ET $(\sim 85.6 \%, \mathrm{p}=0.019)$ with a similar, borderline insignificant trend for survivin, KruskalWallis ANOVA $\mathrm{p}=0.073$. The number of $\mathrm{p} 53$-expressing megakaryocytes in MF was 2 .7-fold greater than in PV trephines, $p=0.0071$. The differences in megakaryocyte positivity between the MPN entities for caspase- 8 and Diablo were not significant, $\mathrm{p}>0.05$ (data not shown).

When apoptotic biomarkers were assessed by mutation status, no significant differences were seen, $p>0.05$. The proportion of p53-expressing megakaryocytes was greater in $C A L R^{\mathrm{Mut}}$ than $J A K 2^{\mathrm{V} 617 \mathrm{~F}+}$ trephines, although this difference was borderline insignificant, Kruskal-Wallis ANOVA $\mathrm{p}=0.0660$, post hoc $\mathrm{p}=0.0667$ (figure 5). Caspase-8-positive and caspase-9-positive megakaryocytes in $J A K 2^{\mathrm{V} 617 \mathrm{~F}+}$ and $C A L R^{\mathrm{Mut}}$ cases were similar and marginally increased when compared with $J A K 2^{\mathrm{V} 617 \mathrm{~F}-} / C A L R^{\mathrm{WT}}$ (figure 5). Mean Diablo and survivin megakaryocyte positivity did not differ by genotype (data not shown).

\section{Apoptotic markers and platelet count}

Since proplatelet production requires megakaryocyte apoptotic signalling, ${ }^{24} 25$ platelet counts were correlated with megakaryocyte positivity for all apoptotic biomarkers in the MPNs. There was a significant, weak positive correlation between caspase- 9 expression and platelet count for all MPNs, $r=0.28, p=0.0018$ (figure 6A). This correlation was strongest for cases of $\mathrm{MF}$, $\mathrm{r}=0.34, \mathrm{p}=0.026$ (figure $6 \mathrm{~B}$ ) and most notably those MPNs with $C A L R^{\mathrm{Mut}}, \mathrm{r}=0.50, \mathrm{p}=0.030$ (figure $6 \mathrm{C}$ ). When cases that had received prior therapy were excluded from this analysis, these correlations remained significant (data not shown). No significant correlations existed between any of the other apoptotic biomarkers and platelet count, $\mathrm{p}>0.05$ (data not shown).

\section{DISCUSSION}

Previous studies have indicated that there is dysfunction of apoptotic mediators in the MPNs, that is, increased antiapoptotic Bcl-XL and reduced pro-death BNIP-3. ${ }^{9}{ }^{13}$ However, the precise apoptotic disruptions promoting abnormal megakaryocyte accumulation and thrombocytosis remain unclear. Here we provide insight into these mechanisms and demonstrate that the enhanced survival of megakaryocytes in the MPNs may be a result of dysregulation of the intrinsic apoptotic pathway. The key protein mediating this survival signature appears to be survivin, an inhibitor of the intrinsic apoptotic cascade. ${ }^{27-29}$ While survivin impedes megakaryocyte death, the reciprocal increase of Diablo, its inhibitor, ${ }^{26}$ is insufficient and unlikely to nullify its antiapoptotic effect. Further, there is low-level upregulation of caspase-9, the main effector of the intrinsic apoptotic cascade (figure 1), ${ }^{16}$ and its expression correlated with platelet count. These data suggest that these aberrations facilitate megakaryocyte hyperplasia and thrombocytosis rather than directing apoptotic execution. The overexpression of pro-death caspase- 8 (extrinsic) and p53 may be an attempt to counteract these changes. The progressive dysfunction of megakaryocyte apoptosis, which has previously shown to be most discernible in MF and CALR-mutated MPNs, ${ }^{9}{ }^{13}$ can now be further supported by marked reductions in pro-death caspase- 9 and accumulation of p53.

Survival advantages gained through inhibition of the intrinsic apoptotic cascade is a key pathological mechanism promoting megakaryocyte hyperplasia in the MPNs. We have previously demonstrated that myeloproliferative megakaryocytes show disrupted expression of the Bcl-2 family of apoptotic biomarkers 


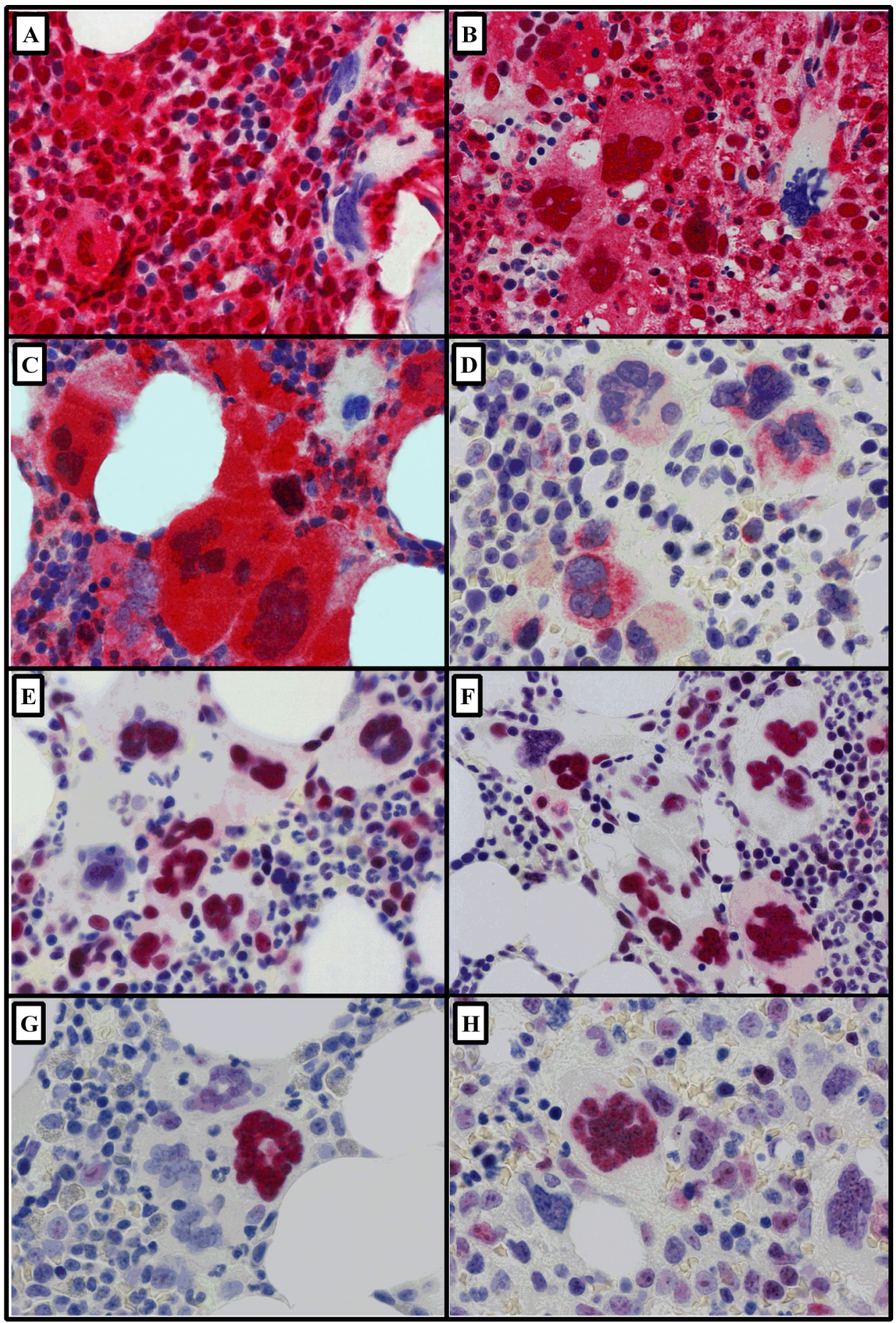

Figure 3 Representative images $(\times 600)$ of immunohistochemically stained megakaryocytes in myeloproliferative neoplasms (bone marrow trephine sections; Fast Red chromogen and haematoxylin counterstain). (A) Caspase-8 in polycythaemia vera and (B) myelofibrosis (MF) and (C) caspase-9 in MF. (D) Diablo-positive megakaryocytes in essential thrombocythaemia (ET). Clusters of megakaryocytes showing strong positive nuclear expression for (E) survivin in ET and (F) in MF. In contrast, nuclear p53 was limited to isolated positive megakaryocytes among a negative majority (G) in $\mathrm{ET}$ and $(\mathrm{H})$ in MF.

(ie, upregulated antiapoptotic Bcl-XL, diminished pro-death BNIP-3) that regulate intrinsic apoptosis. ${ }^{13}$ We further support this theory by showing that survivin is strongly overexpressed in myeloproliferative megakaryocytes. Survivin acts to limit procaspase- 9 cleavage, thereby constraining caspase- 9 activation in megakaryocytes and impeding their death (figure 1). ${ }^{27-29}$ Furthermore, survivin assists megakaryocyte polyploidisation and its increase in the MPNs may be an obligatory survival prerequisite as the majority of these megakaryocytes are of high ploidy (up to $512 \mathrm{~N}$ ). ${ }^{43}{ }^{58}$ Myeloproliferative megakaryocytes attempt to neutralise this survival advantage by expressing Diablo, which directly competes with the antideath effects conferred by excess survivin. ${ }^{26}$ However, a greater proportion of megakaryocytes in both controls $(\sim 26.5 \%)$ and the MPNs $(\sim 19.0 \%)$ expressed survivin than those with upregulated Diablo content. Further, the weak expression intensity of 


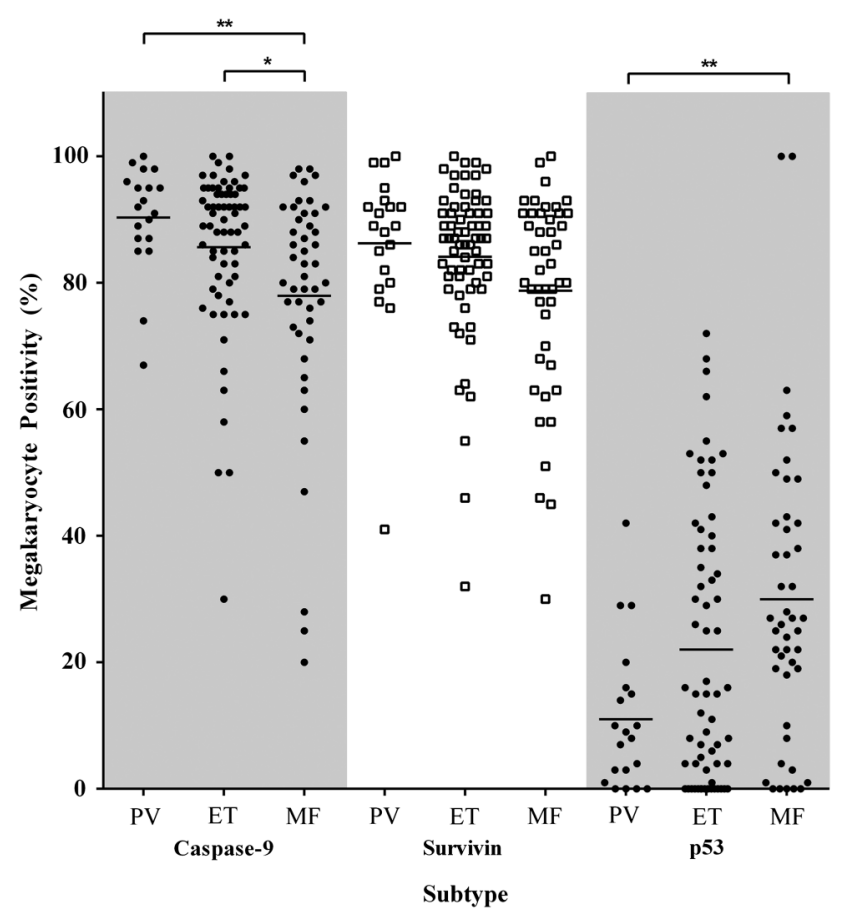

Figure 4 Percentage of megakaryocytes positive according to myeloproliferative neoplasm entity for caspase-9, survivin and p53. Mean megakaryocyte positivity for caspase- 9 was significantly lower in myelofibrosis (MF) than polycythaemia vera (PV) and essential thrombocythaemia (ET), $p=0.0032$ and $p=0.019$, respectively. The per cent positive megakaryocytes in MF was lower for survivin $(p=0.073)$ and higher for p53 than PV and ET, $p=0.0071$ and $p=0.18$, respectively. Statistically significant difference, $p<0.05\left(^{*}\right), p<0.01\left(^{* *}\right)$.

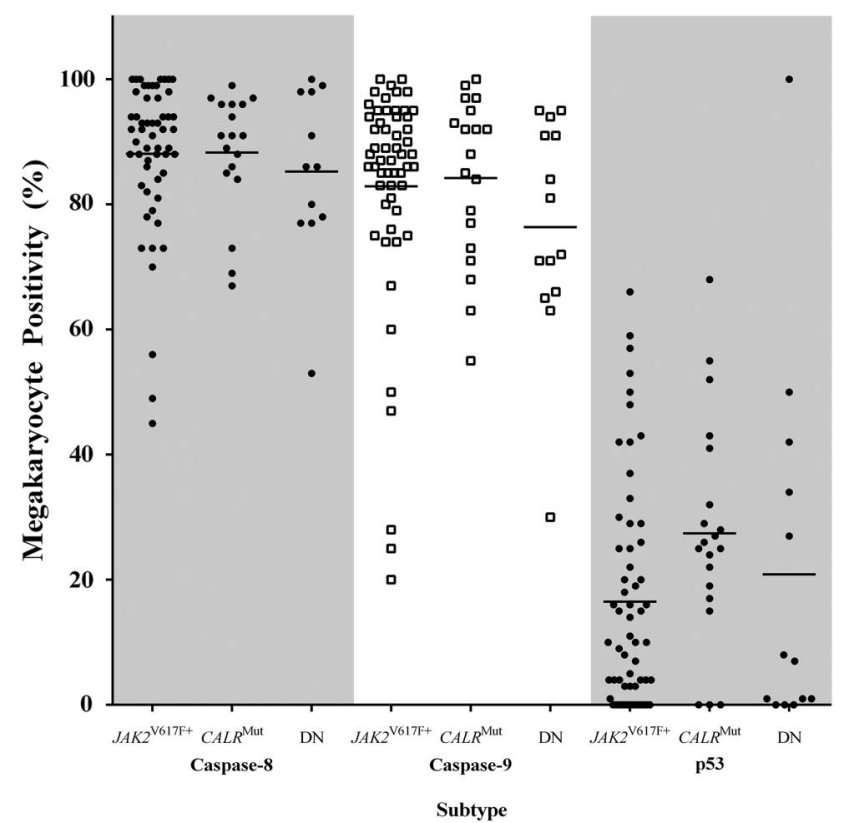

Figure 5 Caspase-8, caspase- 9 and p53 in myeloproliferative neoplasms by $J A K 2^{\mathrm{V} 617 \mathrm{~F}}$ and $C A L R$ mutation status. The percentage of caspase-8-positive and caspase-9-positive megakaryocytes were similar for $J A K 2^{\mathrm{V} 617 F+}$ and $C A L R^{\mathrm{Mut}}$ and not significantly different in comparison to $J A K 2^{\mathrm{V} 617 \mathrm{~F}-} / C A L R^{\mathrm{WT}}$ cases, $\mathrm{p}>0.05$. p53 megakaryocyte positivity was greater in $C A L R^{\text {Mut }}$ than JAK2 $2^{\mathrm{V} 617 \mathrm{~F}+}, \mathrm{p}=0.067$. DN-double negative (ie, JAK2 $2^{\mathrm{V} 167 \mathrm{~F}-} / C A L R^{\mathrm{WT}}$ ).
Diablo-positive megakaryocytes in the MPNs correlates with lower concentrations of the IAP-inhibitor in comparison to stronger survivin signals. This was not seen in control megakaryocytes, where the staining intensity of survivin and Diablo was similar. The survival advantage therefore persists in myeloproliferative megakaryocytes, and in conjunction with other intrinsic apoptotic disturbances is likely to promote their accumulation.

Our data indicate that myeloproliferative megakaryocytes attempt to counteract these survival effects by stimulating the extrinsic apoptotic cascade via caspase-8. This may represent a protective, death-promoting response against megakaryocyte oncogenesis following the accumulation of excess molecular abnormalities. Myeloproliferative megakaryocytes also increase their nuclear p53 content relative to controls where p53 was, and has previously been shown to be, mostly absent. ${ }^{49}$ We postulate that caspase- 8 overactivity induces TP53 gene transcription to produce p53. ${ }^{44}$ p53 then translocates to the megakaryocyte nucleus where it activates the transcription of pro-death biomarkers (eg, Fas, Apaf-1) to stimulate both extrinsic and intrinsic apoptotic cascades. ${ }^{59}$ Moreover, megakaryocytic p53 is potentially increased to directly abrogate the antideath effects of survivin. ${ }^{48}$ Alternatively, this p53 upregulation in myeloproliferative megakaryocytes may be to regulate their polyploidisation by inducing states of megakaryocyte senescence. ${ }^{60}$

There were differences in the megakaryocyte apoptotic profiles according to mutation status. Most notably, CALR-mutated cases showed greater megakaryocytic p53, but not caspase- 8 positivity, than those with the JAK2 ${ }^{\mathrm{V} 617 \mathrm{~F}}$ mutation, although the former was of borderline insignificance. It has previously been shown that calreticulin is involved in caspase- 8 activation. ${ }^{21}$ Our data suggest that the mutated calreticulin gene product does not influence caspase- 8 levels. Rather, it appears that CALR lesions disrupt alternative apoptotic effectors and that affected megakaryocytes will attempt a remedial pro-death response dominated by p53 overexpression. We also show that the small increase in the proportion of caspase-9-positive megakaryocytes in the MPNs is positively correlated with platelet count, and that this correlation strengthens with CALR mutations. We propose two explanations for this data. First, it is likely that CALR lesions interfere indirectly with caspase-9, rather than with caspase-8. Second, the minor increase in caspase- 9 is unlikely to enable megakaryocyte apoptosis to be completed via the intrinsic apoptotic pathway. Rather, several groups have shown that constitutively activated caspase- 9 in the presence of intrinsic antiapoptotic effectors is necessary for proplatelet synthesis and shedding. ${ }^{24}$ 61-63 Therefore, low-level caspase-9 upregulation and concurrent survivin overexpression, especially among CALR-mutated MPNs, may drive thrombocytosis instead of facilitating megakaryocyte apoptosis.

As MPNs progress towards MF, megakaryocytes accumulate additional morphological, topographical and molecular abnormalities, and show the greatest survival signature. ${ }^{7} 1364$ The megakaryocytes in MF also have greater apoptotic disturbances than the other MPNs. This includes lower caspase- 9 and greater numbers of p53-positive megakaryocytes than both PV and ET. It appears that the megakaryocyte attempts to rectify this apoptotic disruption by reducing survivin and overactivating the 'p53-mediated' transcription of its pro-death effectors required for apoptotic execution. ${ }^{59}$ This is consistent with the morphological appearances of megakaryocytes in MF with their greater nuclear pleiomorphism and para-apoptotic changes. ${ }^{7} 1365$ Further, the reduction in number of caspase-9-positive megakaryocytes in MF may be the 

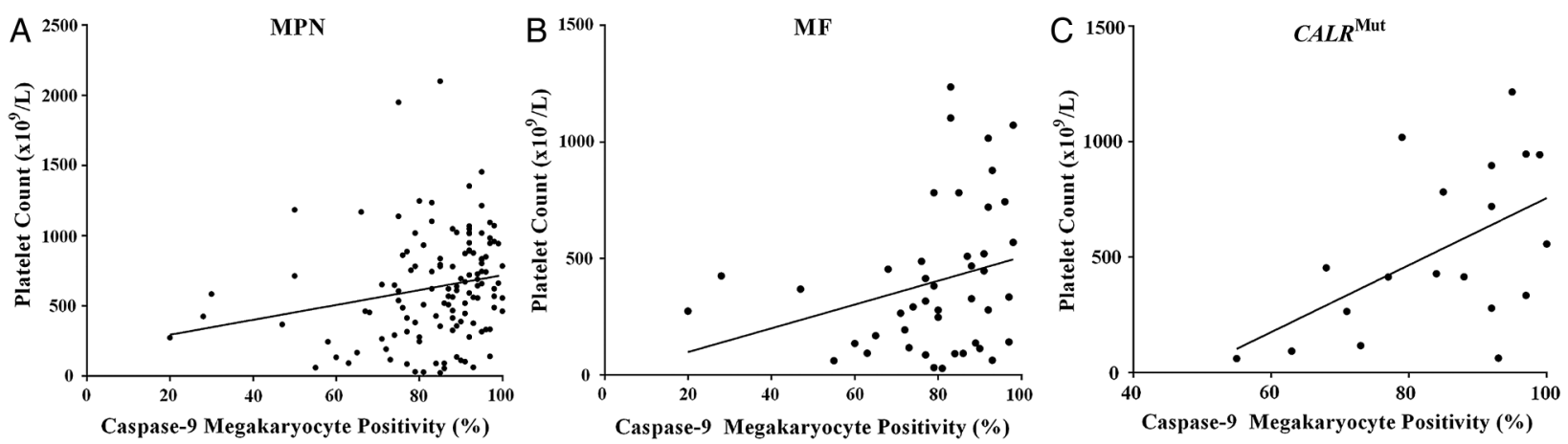

Figure 6 Caspase-9 megakaryocyte positivity correlated significantly with platelet counts for $(A)$ all myeloproliferative neoplasm cases $(r=0.28$, $\mathrm{p}=0.0018)$, (B) myelofibrotic entities $(\mathrm{r}=0.34, \mathrm{p}=0.026)$ and $(\mathrm{C})$ CALR-mutated trephines $(\mathrm{r}=0.50, \mathrm{p}=0.030)$.

mechanism for the lower platelet counts characteristic of this phenotype compared with other MPN entities. ${ }^{766}$

In summary, disruptions targeting the intrinsic apoptotic cascade appear to promote megakaryocyte accumulation and thrombocytosis in the MPNs. Survivin seems to be a key mediator of this antiapoptotic signature and therefore could be a potential therapeutic target. Anti-survivin agents have been developed, which show therapeutic efficacy for many other malignancies. ${ }^{67-69}$ As such, targeting survivin in the MPNs could potentially control megakaryocyte accumulation and downstream effects (eg, stromal accumulation). Further, a synergistic option could potentially be achieved by including Diablo/ Smac-like mimetics ${ }^{70-72}$ to augment innate and already upregulated megakaryocytic Diablo in the MPNs.

\section{Take home messages}

- Dysregulation of intrinsic apoptotic mechanisms in megakaryocytes in the myeloproliferative neoplasms (MPNs) promotes their hyperplasia and thrombocytosis.

- Survivin, a protein that inhibits the intrinsic pathway of apoptosis, appears to be a key mediator of apoptotic failure of megakaryocytes in the MPNs.

- Caspase-9 dysfunction may explain the heightened thrombocytosis in MPNs, especially among those with CALR mutations.

\section{Handling editor Mary Frances McMullin}

Acknowledgements JAJM received financial support from the Royal College of Pathologists of Australasia through the RCPA Scholarship in Pathology for Medical Schools 2015 for this study.

Contributors JAJM, KAF and BM performed the immunohistochemical studies. C-CS, H-WI and BBG conducted the molecular analyses. JAJM, KAF, SK, BM and WNE performed the megakaryocyte enumeration for all cases. JAJM conducted the statistical analyses. C-CS, HWI, CF and WNE undertook the morphological review. $\mathrm{RH}$ and WNE initiated the study. All authors contributed to the writing of the manuscript.

Funding The study was supported by the Australian Leukaemia and Lymphoma Group and Novartis Pharmaceuticals Australia Pty Ltd, the Cancer Council Western Australia, Perpetual Foundation-Ann Helene Toakley Charitable Endowment and the Royal College of Pathologists of Australasia.

Competing interests None declared.

Patient consent Obtained.

Ethics approval (1) Sir Charles Gairdner Hospital Human Research Ethics Committee (\#2012-094). (2) The University of Western Australia Human Research
Ethics Committee (\#RA/4/1/6566). (3) The Institutional Review Board of the University of Hong Kong/Hospital Authority Hong Kong West Cluster (\#UW 13-189).

Provenance and peer review Not commissioned; externally peer reviewed.

Data sharing statement Data are available upon request and at the discretion of the authors.

Open Access This is an Open Access article distributed in accordance with the Creative Commons Attribution Non Commercial (CC BY-NC 4.0) license, which permits others to distribute, remix, adapt, build upon this work non-commercially, and license their derivative works on different terms, provided the original work is properly cited and the use is non-commercial. See: http://creativecommons.org/ licenses/by-nc/4.0/

\section{REFERENCES}

1 James C, Ugo V, Le Couédic JP, et al. A unique clonal JAK2 mutation leading to constitutive signalling causes polycythaemia vera. Nature 2005;434:1144-8.

2 Levine RL, Wadleigh $\mathrm{M}$, Cools J, et al. Activating mutation in the tyrosine kinase JAK2 in polycythemia vera, essential thrombocythemia, and myeloid metaplasia with myelofibrosis. Cancer Cell 2005;7:387-97.

3 Baxter EJ, Scott LM, Campbell PJ, et al. Acquired mutation of the tyrosine kinase JAK2 in human myeloproliferative disorders. Lancet 2005;365:1054-61.

4 Klampfl T, Gisslinger H, Harutyunyan AS, et al. Somatic mutations of calreticulin in myeloproliferative neoplasms. N Engl J Med 2013;369:2379-90.

5 Nangalia J, Massie CE, Baxter EJ, et al. Somatic CALR mutations in myeloproliferative neoplasms with nonmutated JAK2. N Engl J Med 2013;369:2391-405.

6 Tefferi A, Thiele J, Orazi A, et al. Proposals and rationale for revision of the World Health Organization diagnostic criteria for polycythemia vera, essential thrombocythemia, and primary myelofibrosis: recommendations from an ad hoc international expert panel. Blood 2007;110:1092-7.

7 Swerdlow SH, Campo E, Harris NL, et al. WHO classification of tumours, Volume 2. 4th edn. Geneva: WHO, 2008; WHO classification of tumours of haematopoietic and lymphoid tissues.

8 Tefferi A, Vainchenker W. Myeloproliferative neoplasms: molecular pathophysiology, essential clinical understanding, and treatment strategies. J Clin Oncol 2011;29:573-82.

9 Florena AM, Tripodo C, Di Bernardo A, et al. Different immunophenotypical apoptotic profiles characterise megakaryocytes of essential thrombocythaemia and primary myelofibrosis. J Clin Pathol 2009;62:331-8.

10 Tognon R, Gasparotto EPL, Leroy JMG, et al. Differential expression of apoptosis-related genes from death receptor pathway in chronic myeloproliferative diseases. J Clin Pathol 2011;64:75-82.

11 Treliński J, Chojnowski K, Cebula-Obrzut B, et al. Impaired apoptosis of megakaryocytes and bone marrow mononuclear cells in essential thrombocythemia: correlation with JAK2V617F mutational status and cytoreductive therapy. Med Oncol 2012;29:2388-95.

12 Koopmans SM, Schouten HC, van Marion AM. Anti-apoptotic pathways in bone marrow and megakaryocytes in myeloproliferative neoplasia. Pathobiology 2014;81:60-8.

13 Malherbe JAJ, Fuller KA, Arshad A, et al. Megakaryocytic hyperplasia is driven by disordered proliferative, apoptotic and epigenetic mechanisms. J Clin Path 2016;62:155-63

14 Ghavami S, Hashemi M, Ande SR, et al. Apoptosis and cancer: mutations within caspase genes. J Med Genet 2009;46:497-510.

15 Olsson M, Zhivotovsky B. Caspases and cancer. Cell Death Differ 2011;18:1441-9.

16 Ouyang L, Shi Z, Zhao S, et al. Programmed cell death pathways in cancer: a review of apoptosis, autophagy and programmed necrosis. Cell Proliferat 2012;45:487-98. 
17 Hakem A, El Ghamrasni S, Maire G, et al. Caspase-8 is essential for maintaining chromosomal stability and suppressing B-cell lymphomagenesis. Blood 2012;119:3495-502.

18 Liu JR, Opipari AW, Tan L, et al. Dysfunctional apoptosome activation in ovarian cancer: implications for chemoresistance. Cancer Res 2002;62:924-31.

19 Park JY, Park JM, Jang JS, et al. Caspase 9 promoter polymorphisms and risk of primary lung cancer. Hum Mol Genet 2006;15:1963-71.

20 Gangwar R, Mandhani A, Mittal RD. Caspase 9 and caspase 8 gene polymorphisms and susceptibility to bladder cancer in north Indian population. Ann Surg Oncol 2009:16:2028-34.

21 Wang W, Groenendyk J, Michalak M. Calreticulin signaling in health and disease. Int J Biochem Cell B 2012:44:842-6.

22 Vannucchi AM, Rotunno G, Bartalucci N, et al. Calreticulin mutation-specific immunostaining in myeloproliferative neoplasms: pathogenetic insight and diagnostic value. Leukemia 2014;28:1181-8.

23 Stein H, Bob R, Dürkop H, et al. A new monoclonal antibody (CAL2) detects CALRETICULIN mutations in formalin-fixed and paraffin-embedded bone marrow biopsies. Leukaemia 2016;30:131-5.

24 De Botton S, Sabri S, Daugas $E$, et al. Platelet formation is the consequence of caspase activation within megakaryocytes. Blood 2002;100:1310-17.

25 White MJ, Schoenwaelder SM, Josefsson EC, et al. Caspase-9 mediates the apoptotic death of megakaryocytes and platelets, but is dispensable for their generation and function. Blood 2012;119:4283-90.

26 Chai J, Du C, Wu JW, et al. Structural and biochemical basis of apoptotic activation by Smac/DIABLO. Nature 2000;406:855-62.

27 Dohi T, Beltrami E, Wall NR, et al. Mitochondrial survivin inhibits apoptosis and promotes tumorigenesis. J Clin Invest 2004;114:1117-27.

28 Sah NK, Khan Z, Khan GJ, et al. Structural, functional and therapeutic biology of survivin. Cancer Lett 2006;244:164-71.

29 Duffy MJ, O'Donovan N, Brennan DJ, et al. Survivin: a promising tumor biomarker. Cancer Lett 2007;249:49-60.

30 Ren Y, Akyurek N, Schlette E, et al. Expression of Smac/DIABLO in B-cell non-Hodgkin and Hodgkin lymphomas. Hum Pathol 2006;37:1407-13.

31 Pluta A, Wrzesien-Kus A, Cebula-Obrzut B, et al. Influence of high expression of Smac/DIABLO protein on the clinical outcome in acute myeloid leukemia patients. Leuk Res 2010;34:1308-13.

32 Mansour A, Nabil M, Ali-Labib R, et al. Reciprocal expression of survivin and SMAC/DIABLO in primary breast cancer. Med Oncol 2012;29:2535-42.

33 Fulda S. Inhibitor of apoptosis (IAP) proteins in haematological malignancies: molecular mechanisms and therapeutic opportunities. Leukemia 2014;28:1414-22.

34 Shinozawa I, Inokuchi K, Wakabayashi I, et al. Disturbed expression of the anti-apoptosis gene, survivin, and EPR-1 in hematological malignancies. Leukemia Res 2000;24:965-70.

35 Mori $A$, Wada $H$, Nishimura $Y$, et al. Expression of the antiapoptosis gene survivin in human leukemia. Int J Hematol 2002;75:161-5.

36 Nakagawa $Y$, Yamaguchi $S$, Hasegawa $M$, et al. Differential expression of survivin in bone marrow cells from patients with acute lymphocytic leukemia and chronic lymphocytic leukemia. Leukemia Res 2004;28:487-94.

37 Small S, Keerthivasan G, Huang Z, et al. Overexpression of survivin initiates hematologic malignancies in vivo. Leukemia 2010;24:1920-6.

38 Adida C, Haioun C, Gaulard P, et al. Prognostic significance of survivin expression in diffuse large B-cell lymphomas. Blood 2000;96:1921-5.

39 Ansell SM, Arendt BK, Grote DM, et al. Inhibition of survivin expression suppresses the growth of aggressive non-Hodgkin's lymphoma. Leukemia 2004;18:616-23.

40 Martinez A, Bellosillo B, Bosch F, et al. Nuclear survivin expression in mantle cell lymphoma is associated with cell proliferation and survival. Am J Pathol 2004;164:501-10.

41 Schlette EJ, Medeiros LJ, Goy A, et al. Survivin expression predicts poorer prognosis in anaplastic large-cell lymphoma. J Clin Oncol 2004;22:1682-8.

42 Wen Q, Leung C, Huang Z, et al. Survivin is not required for the endomitotic cell cycle of megakaryocytes. Blood 2009;114:153-6.

43 McCrann DJ, Ravid K. Survivin localization during endomitosis of high ploidy mouse megakaryocytes. Blood 2010;116:2192-3.

44 Schuler M, Bossy-Wetzel E, Goldstein JC, et al. p53 induces apoptosis by caspase activation through mitochondrial cytochrome c release. J Biol Chem 2000;275:7337-42.

45 Chipuk JE, Green DR. Dissecting p53-dependent apoptosis. Cell Death Differ 2006;13:994-1002

46 Tomita Y, Marchenko N, Erster S, et al. WT p53, but not tumor-derived mutants, bind to $\mathrm{BCl} 2$ via the DNA binding domain and induce mitochondrial permeabilization. J Biol Chem 2006;281:8600-6.
47 Deng X, Gao F, Flagg T, et al. Bcl2's flexible loop domain regulates p53 binding and survival. Mol Cell Biol 2006;26:4421-34.

48 Mirza A, McGuirk M, Hockenberry TN, et al. Human survivin is negatively regulated by wild type p53 and participate in p53-dependent apoptotic pathway. Oncogene 2002;21:2613-22.

49 Datta NS, Long MW. Modulation of MDM2/p53 and cyclin-activating kinase during the megakaryocyte differentiation of human erythroleukemia cells. Exp Hematol 2002:30:158-65

50 Horie K, Kubo K, Yonezawa M. p53 dependency of radioadaptive responses in spleen colonies and peripheral blood-cell counts in C57BL mice. J Radiat Res 2002:43:353-60

51 Mahdi T, Brizard A, Millet C, et al. In vitro p53 and/or Rb antisense oligonucleotide treatment in association with growth factors induces the proliferation of peripheral hematopoietic progenitors. J Cell Sci 1995;108(Pt 3):1287-93.

52 Ritchie A, Vadhan-Raj S, Broxmeyer HE. Thrombopoietin suppresses apoptosis and behaves as a survival factor for the human growth factor-dependent cell line, M07e. Stem Cells 1996;14:330-6.

53 Apostolidis PA, Woulfe DS, Chavez M, et al. Role of tumor suppressor p53 in megakaryopoiesis and platelet function. Exp Hematol 2012;40:131-42.

54 Apostolidis PA, Lindsey S, Miller WM, et al. Proposed megakaryocytic regulon of p53: the genes engaged to control cell cycle and apoptosis during megakaryocytic differentiation. Physiol Genomics 2012;44:638-50.

55 Harutyunyan A, Klampfl T, Cazzola M, et al. p53 lesions in leukemic transformation. N Engl J Med 2011;364:488-90.

56 Raza S, Viswanatha D, Frederick L, et al. TP53 mutations and polymorphisms in primary myelofibrosis. Am J Hematol 2012;87:204-6.

57 Greaves WO, Verma S, Bisrat T, et al. TP53 mutation is rare in primary myelofibrosis. Leuk Lymphoma 2013;54:1552.

58 Mazur EM, Lindquist DL, de Alarcon PA, et al. Evaluation of bone marrow megakaryocyte ploidy distributions in persons with normal and abnormal platelet counts. J Clin Lab Med 1988;111:194-202.

59 Yu J, Zhang L. The transcriptional targets of p53 in apoptosis control. Biochem Bioph Res Co 2005;331:851-8.

60 Fuhrken PG, Apostolidis PA, Lindsey S, et al. Tumor suppressor protein p53 regulates megakaryocytic polyploidization and apoptosis. J Biol Chem 2008;283:15589-600.

61 White MJ, Kile BT. Apoptotic processes in megakaryocytes and platelets. Semin Hematol 2010;47:227-34.

62 Josefsson EC, James C, Henley KJ, et al. Megakaryocytes possess a functional intrinsic apoptosis pathway that must be restrained to survive and produce platelets. J Exp Med 2011;208:2017-31.

63 Debrincat MA, Josefsson EC, James $\mathrm{C}$, et al. Mcl-1 and $\mathrm{BCl}-\mathrm{xL}$ coordinately regulate megakaryocyte survival. Blood 2012;119:5850-8.

64 Barosi G, Rosti V, Bonetti $\mathrm{E}$, et al. Evidence that prefibrotic myelofibrosis is aligned along a clinical and biological continuum featuring primary myelofibrosis. PLOS ONE 2012; 7:e35631.

65 Asher E, Payne CM, Berstein C. Evaluation of cell death in EBV-transformed lymphocytes using agarose gel electrophoresis, light microscopy and electron microscopy: II. Induction of non-classic apoptosis ("para-apoptosis") by tritiated thymidine. Leuk Lymphoma 1995;19:107-19.

66 Gangat N, Caramazza D, Vaidya R, et al. DIPSS plus: a refined dynamic international prognostic scoring system for primary myelofibrosis that incorporates prognostic information from karyotype, platelet count and transfusion status. J Clin Oncol 2011;29:392-7.

67 Weiss A, Brill B, Borghouts C, et al. Survivin inhibition by an interacting recombinant peptide, derived from the human ferritin heavy chain, impedes tumor cell growth. J Cancer Res Clin Oncol 2012;138:1205-20.

68 Groner B, Weiss A. Targeting survivin in cancer: novel drug development approaches. BioDrugs 2014;28:27-39.

69 Mehta A, Zhang L, Boufraqech M, et al. Inhibition of survivin with YM155 induces durable tumour response in anaplastic thyroid cancer. Cancer Clin Res 2015;21:4213-32.

70 Runckel KL, Skitzki J, Hernandez F, et al. The SMAC mimetic LCl-161 augments the in vitro and in vivo anti-tumour activity of rituximab and chemotherapy in rituximab relapse/refractory lymphoma models. Blood 2014;124:3110.

71 Chromik J, Safferthal C, Serve H, et al. Smac mimetic primes apoptosis-resistant acute myeloid leukaemia cells for cytarabine-induced cell death by triggering necroptosis. Cancer Lett 2014;344:101-9.

72 Amaravadi RK, Schilder RJ, Martin LP, et al. A phase I study of the SMAC-mimetic birinapant in adults with refractory solid tumors or lymphoma. Molec Cancer Ther 2015;14:2569-75. 